\title{
Intervention strategies with 2D cellular automata for testing SARS-CoV-2 and reopening the economy
}

Igor Lugo ( $\nabla$ igorlugo@crim.unam.mx )

National Autonomous University of Mexico https://orcid.org/0000-0003-3490-2654

Martha G. Alatriste-Contreras

National Autonomous University of Mexico https://orcid.org/0000-0001-5549-4747

\section{Research Article}

Keywords: Intervention strategy, SARS-CoV-2, 2D cellular automaton

Posted Date: July 9th, 2020

DOI: https://doi.org/10.21203/rs.3.rs-40739/v1

License: (9) This work is licensed under a Creative Commons Attribution 4.0 International License. Read Full License

Version of Record: A version of this preprint was published at Scientific Reports on August 5th, 2022. See the published version at https://doi.org/10.1038/s41598-022-17665-3. 


\title{
Intervention strategies with 2D cellular automata for testing SARS-CoV-2 and reopening the economy
}

\author{
Igor Lugo ${ }^{1,2, *,+}$ and Martha G. Alatriste-Contreras ${ }^{3,+}$ \\ ${ }^{1}$ National Autonomous University of Mexico, Centro Regional de Investigaciones Multidisciplinarias (CRIM), \\ Cuernavaca, Mor., 62210, Mexico \\ ${ }^{2}$ National Autonomous University of Mexico, Centro de Ciencias de la Complejidad (C3), Mexico City, 04510, Mexico \\ ${ }^{3}$ National Autonomous University of Mexico, Facultad de Economía, Mexico City, 04510, Mexico \\ *igorlugo@crim.unam.mx \\ +these authors contributed equally to this work
}

\begin{abstract}
During the period of time between a new disease outbreaks and its vaccine is deployed, the health and the economic systems have to find a testing strategy for reopening activities. In particular, asymptomatic individuals, who transmit locally the covid-19 indoors, have to be identified and isolated. We proposed a 2D cellular automaton based on the SI epidemic model for selecting the most desirable testing frequency and identifying the best fitting size of random trails on local urban environments to diagnose SARS-CoV-2 and isolate infected people. We used the complex systems approach to face the challenge of a large-scale test strategy based on urban interventions, starting with first responders and essential workers. We used the case of Mexico to exemplify a credible and intelligent intervention that reduces the virus transmission and detects economic and health costs. Findings suggest that controlling and stopping the virus transmission in a short period of time are possible if the frequency of testing is daily and the percentage of random samples to be tested is at least $90 \%$. This combination of model parameters represents the least expensive intervention compared to others. Therefore, the key for a national testing-isolating strategy is local interventions.
\end{abstract}

\section{Introduction}

In the current pandemic of the coronavirus disease 2019 (covid-19), the health and the economic systems of all nations have been stressed to their limits and started to collapse. This situation has showed the vulnerability and fragility of such systems to expected external shocks ${ }^{1-3}$. The economic activity in each country has decreased due to the coronavirus restrictions, in particular the national lockdowns. It slowed the severe acute respiratory syndrome coronavirus 2 (SARS-CoV-2) transmission, but affected the consumer behavior thus decreasing demand, which, as consequence, slowed down or stopped the flow of resources in the supply chain. The main expected effect has been the increasing unemployment. For example, in the USA the unemployment has showed an amount of 21.0 millions American jobs lost, a rate of 13.3\% in May ${ }^{4}$. In Mexico, what is even worse is the lack of accurate data about the impact of such a lockdown in the economy. According to the Occupation and Employment Survey (ETOE in Spanish) of the National Institute of Statistics and Geography (INEGI in Spanish), from March to April 2020, the unemployment increased 12.5 millions $^{5}$. Therefore, the economic conditions point out the importance to open the economy as soon as possible, but it presents a higher health cost to the population. In particular, opening the economy without a vaccine presents a social dilemma between the economy vs the health of the nation. This idea was clearly stated by Anthony Fauci, NIH National Institute of Allergy and Infectious Diseases Director, who said "How many deaths and how much suffering are you willing to accept to get back to what you want to be some form of normality sooner rather than later?"6 Days before this statement, important ideas came from the Nobel Laureate economist Paul Romer", who stated "mass testing is the key to reopening the economy", and, later on, Osterholm ${ }^{8}$ and his colleague of the CIDRAP proposed a smart testing framework. Then, such a dilemma is solved based on a sequential testing and isolating strategies that can identify and lock down those who are infected, in particular asymptomatic individuals. Therefore, we proposed a theoretical approximation based on cellular automata (CA) and the Susceptible-Infectious (SI) epidemic models to explore local testing scenarios—small workplaces and communities-for returning to work and keeping slow the contagion. Local strategies are the most appropriate interventions for identifying asymptomatic individuals and keeping track of the health and the economic costs.

We used the complex systems approximation based on the epidemiology and the economics fields to explore testing scenarios. These scenarios consider the reverse transcriptional loop-mediated isothermal amplification (RT-LAMP) viral test, proposed by Mexican and other scientists, that identifies the current infection in a period of $15 \min ^{10,11}$. However, there are other test options- the antibody test, computed tomography (CT) scans, and reverse-transcriptase polymerase chain reaction 
(RT-PCR) viral test-that diagnose SARS-CoV-2. Based on such a viral test or similar tests in the market and the well-known 2D CA models ${ }^{12-14}$, we applied the discrete, spatial formulation of the SI model because it describes the simplest form of contagion without immunity, treatment, and vaccine ${ }^{15-17}$. Then, we identified the time interval to apply the covid-19 test and to select a subset of randomly chosen individuals for testing into local environments—i.e., hospitals, companies, academic departments, or similar others. Even though our approximation is easily generalized to other countries due to its vital importance, we used the case of Mexico because we are aware of the unsafe health conditions when reopening the economy without a vaccine and the flawed interventions to face the national health crisis. To date (July 1, 2020), Mexico has showed an increasing trend in confirmed cases and deaths of covid-19, and the federal interventions do not consider a testing strategy to control and stop the virus transmission as one of its main goals ${ }^{18-20}$. However, in May 31, 2020, the federal government restarted the economy activity in the country. Therefore, it is evident that without a vaccine in the short term, people of Mexico, particularly in urban areas, will be exposed to a higher risk to get SARS-CoV-2 and, hence, worsen the economic activity.

This study focuses on showing a simple theoretical, ${ }^{21}$ local model to understand and to plan the best strategy to restart activities taking care of the health of people and considering the economic costs. In particular, during the time period between the spread of the disease and the production and distribution of its vaccine, we proposed an action plan to support and guide the health and the economic systems. Our main goals are to control and stop the virus transmission before all people get infected and to identify the economic cost of the intervention. We are interested in answering the following three questions: how to operationalize the return to work processes keeping the contagious rate lower? What is the best and the worst scenarios to open the economy? And what are their economic costs and benefits? To answer these questions, we followed Romer ${ }^{7}$ and Osterholm ${ }^{8}$ ideas in which different testing situations have to be analyzed to identify the best combination between the frequency of testing and the random sampling for selecting individuals. Base on this information, we used an exploratory data analysis for identifying trends and correlations to describe the best strategies to open the economy. In particular, we used a sensitivity analysis based on the sampling-based and scatterplot approaches. To develop these analyses, we used the Python programming language to generate and analyze data, and we share it based on the open science principles ${ }^{22}$. We strongly believe that the testing capacity and its organization are the keys to reopen the economy and reduce the risk of contagion. We investigate the hypothesis that the scale of the community or workplace matters to reduce the time steps of transmission and to stop the spread of SARS-CoV-2. A small group of people concentrated indoors can be monitored and, consequently, the disease transmission can be better controlled in a few time steps, if the frequency of testing is daily and the size of random trails is at least $70 \%$ of the people. On the other hand, large groups of people partially concentrated indoors control worse the disease transmission in large time steps, if the frequency of testing is greater or equal than twice a week and the size of random trails is less than $70 \%$ of the people. The former is less expensive than the latter because the testing is intensive in time periods. In line with Prather et. al. ${ }^{23}$, Zhang et al., ${ }^{24}$, and Dbouka, T. and Drikakisb ${ }^{25}$, we believe that, even though people follow health prevention measures to not get sick-i.e., social distancing and face cover-and the official guidelines to reopen the economy-i.e., flexible work hours and cleaning continuously surfaces, equipment, and other elements of work environment - asymptomatic individuals can infect a group of people concentrated indoors during a small time period of a day because of the airborne transmission. Therefore, small groups of first responders-workers related to the healthcare, transportation, cleaning, and food service systems - have to be tested and monitored constantly to guarantee the basic services to population. Eventually, other essential workers — such as professors and researchers in academic places-have to be tested as well. Finally, testing to everyone based on local communities.

This paper contributes to the scientific efforts for controlling the current pandemic of the SARS-CoV-2 and preparing us for the next pandemics to come. Identifying the frequency of testing and the random sample to be tested based on the size of local establishments, we can generate an effective, credible, and intelligent approach to save lives and return to work safely. We have to consider this type of approaches using the available tests until a vaccine is approved and deployed to everyone. Therefore, it is fundamental a testing strategy based on local environments, which are more accessible to coordinate efforts in the shortest time possible, to auto-organized the society fighting against the covid-19, and to reopen the economy.

This paper is organized as follows: Material section describes the basic parameters to use in the 2D CA model; the Method section describes the CA based on the SI model and the testing-isolating rule; the Result section shows findings and their interpretations; finally, the Discussion section shows final remarks and suggestions.

\section{Materials}

Since our study shows a theoretical approximation for identifying the intervention related to testing SARS-CoV-2 and reopening safely the economy, we use particular types of data for contextualizing and calibrating our 2D CA. In particular, we selected a particular rate of transmission, a viral test, and a randomized controlled trails (RCTs) approximation.

Firstly, we use the rate of transmission - the number of secondary infections from one infected individual-between 2 to 2.5 provided by the Mexican federal government and the Pan American Health Organization (OPS in Spanish) ${ }^{26}$. Next, we assume the use of a viral test that diagnoses SARS-CoV-2 around a period between 15 minutes or less in the same day of 
testing, and we suppose that the test shows $0 \%$ of false positives. In particular, we are interested in the RT-LAMP viral test. We assumed a price per test from $\$ 1200$ to $\$ 7500 \mathrm{mxn}-$ from $\$ 53$ to $\$ 332$ usd $^{27}$. Finally, we use the randomized controlled trails (RCTs) approximation in our model to show its importance and practical implementation in local indoor environments for reducing the virus transmission ${ }^{28}$. For example, different sizes of workplaces, based on the number of workers indoors, can randomly choose individuals and keep track of their testing results and contact tracing to monitor data. Therefore, because of the scale of the analysis, our approach can identify the benefits and costs of the testing-isolating strategy. Benefits are in terms of the number of days for reducing or stopping the virus transmission and for implementing the strategy. Costs are in terms of monetary values and health disadvantages or infected people when implementing the strategy.

In addition, we used a second type of materials based on programming libraries. We applied the Python programming language using the following libraries: Matplotlib, Numpy, Scipy, random, and pickle. We provide the programing code, the data, the 2D CA, and the results in our Open Science Framework (OSF) project (see Local testing SARS-CoV-2 strategies and reopening the economy).

\section{Method}

Despite the fact that there are different epidemic models ${ }^{29-31}$, the SI formulation describes the spread of infectious diseases without considering immunity, treatment, and vaccine. These characteristics are closely related to the current knowledge of the SARS-CoV-2 behavior ${ }^{24}$. The SI model is defined as followed:

$$
\begin{aligned}
& \frac{d S}{d t}=\frac{-\beta S I}{N} \\
& \frac{d I}{d t}=\beta I\left(1-\frac{I}{N}\right)
\end{aligned}
$$

where $\mathrm{S}=$ susceptible, $\mathrm{I}=$ infected; $S+I=N$ is the constant population size of individuals in the model; and $\beta=$ contagious rate between $S$ and $I$. Once $S$ is changed to $I$, without a treatment, individuals stay infected. Its dynamic shows an exponential growth of $I$, meanwhile $S$ shows an inverse shape of $I$. Therefore, this formulation shows a mathematical tractability advantage due to its continuous approximation ${ }^{16}$.

Based on this formulation, we used the discrete, spatial approximation based on a 2D CA because it can describe accurately the local indoors contagious process. This type of modeling has been applied not only to epidemics, but also to describe large-scale processes, such as the growth of cities ${ }^{14}$. Therefore, we used the epidemiology concepts of the SI model to describe the method and to interpret results, but we used the framework of the complex systems to build and contextualize the 2D CA.

In this section, we present the 2D CA baseline model in which its rules are formed by deterministic and stochastic transitional rules. The former rule specifies the contagious mechanism based on the rate of transmission. The latter rule describes the testing-isolating strategy to control the virus transmission. These rules identify how the scale-the total number of cells in the $\mathrm{CA}$ - affects the intervention strategy: the testing-isolating frequencies and the number of cells randomly selected to be tested. Next, we apply a sensitivity analysis sampling-based and scatterplot approaches changing the parameters related to scale, test frequency, and the number of people randomly chosen for testing. The resulted scatterplots are analyzed by the Spearman rank coefficient to test the sensitivity of the selected parameters. Finally, we identify the statistical distribution of total number of people tested per model specification to provide the economic cost of the intervention strategy. Those distributions are identified by using the Kolmogorov-Smirnov (KS) goodness-of-fit test ${ }^{32}$.

\section{Baseline CA}

We proposed a model using a regular 2D tessellation as a grid, where the state of each cell $i$ depends on three attributes: Susceptible (S), Infected (I), and Tested-Isolated (TI). Then, each cell has three states, $S_{i}=0, I_{i}=1$, and $T I_{i}=2$. We selected the Moore neighborhood $\Omega_{i}$ defined by eight adjacent cells. The initial condition at time $t=0$ are the following:

$$
I_{i}=1, \quad S_{i}=0, \quad \text { and } \quad T I_{i}=0
$$

where $I_{i}=1$ is located in the center of the array. In this model, every $t$, which is an iteration, describes a day. Then, the transitional rules at any time $t$ are the following:

$$
\begin{aligned}
& \text { if } \quad S_{i}(t)=0 \quad \text { and } \quad \sum_{k \subset \Omega_{i}} I_{i}(t)=1 \quad \text { or }=2 \quad \text { or }=3 \\
& \text { then } I_{i}(t+1)=1
\end{aligned}
$$


where equation (4) describes the rate of transmission based on the number of susceptible receptors in the neighborhood. We used from one to three active cells in the neighborhood as the unweighted infectious rate. Therefore, one infected cell can transmit the virus to its neighbors, from $S_{i}=0$ to $I_{i}=1$. Next, we defined the testing-isolating rule that depends on specific time frequencies (intervals) $t_{\text {interval }}$ and a random sample of cells to detect and isolate infected cells.

$$
\begin{aligned}
& \text { If } t=t_{\text {interval }}: \\
& \text { random_sample }=N \phi \\
& \text { For } i \text { in random_sample: } \\
& \quad \text { If } I_{i}(t)=1 \text { then } T I_{i}(t+1)=2
\end{aligned}
$$

where $t_{\text {interval }}$ describes three types of values $\{1=$ daily, $3=$ twice a week, and $7=$ a week $\}$, and random_sample is the fraction of the population selected randomly_based on a uniform distribution-by $\phi$ to be tested. Then, if the cell is detected as infected $I$ in $t+1$ it is isolated. This type of cell stays isolated throughout its life, i.e., in next iterations such cells were not considered in the computation. Then, in each $t_{\text {interval }}$, the number of cells associated with the random sample decreases because of the number of isolated cells. Therefore, the number of testing cells is collected to identify the total economic cost per model.

Next, we applied a sensitivity analysis based on scatterplots to show different scenarios when varying the size, testingisolating intervals, and the percentage of people in the sample. In particular, we are searching for possible correlations between the testing intervals and the size of random samples that identify the best combination of parameters to reduce or stop the virus transmission in fewer time steps. We analyzed and selected the scenarios of interest that best describe the relationship between those parameters. Moreover, these resulted scatterplots are analyzed by the Spearman rank correlation, and we identify the statistical distribution associated with the total number of cells that were tested in each combination of model parameters. To identify the best fit of statistical distributions, we used the KS goodness-of-fit test.

\section{Results}

The following results are based on the assumption mentioned earlier that, even though persons follow the infection prevention and official guidelines for reopening the economy, the transmission of the virus is highly probable by spreading in aerosol and droplets. We analyzed the case when one asymptomatic person spreads the virus indoors in several days. For the sake of simplicity and accuracy, results of the sensitivity analysis show three combinations of parameters based on three different sizes of array. This set of combinations was selected because the other possible combinations showed a complete contagion of cells (see the OSF project for the full set of results). These are scenarios that we want to avoid due to their higher health cost. Therefore, the following results summarize and describe accurately our main ideas about an intervention based on local testing strategies.

Initially, we present the effect of the virus transmission without any testing-isolating strategy in local indoor environments (Figure 1). The spread of the virus depends on the number of cells in the array. After few time steps, all cells were infected. This relationship shows an increased curve with a positive trend. Therefore, small local indoor environments transmit faster the virus. On the other hand, large local indoor environments transmit the virus slower. At the end, people in both environments get totally infected.

In addition, we present one execution of our CA model with particular parameters to exemplify the performance of our code (Figure 2). Figure 2 displays six frames that describe the dynamics of the CA. We observe in $t_{5}$ that the symmetry of the virus spreading was broken when the testing-isolating rule is introduced. As we mentioned in the rule (5), the random sampling instruction generates different transmission patterns in the model dynamics. Then, every time step when this rule is applied, we try to control or stop the virus transmission pattern identifying infected cells and isolate them. This goal is achieved when the infected cells were zero, in $t_{37}$. This is displayed in subfigure 2(a) that shows the dynamics of the susceptible, infected and tested-isolated cells. Therefore, this simulation shows the stop of the virus transmission before all cells get infected in larger time steps.

Next, we show findings associated with the sensitivity analysis. Because of the computational expenses of executing the model 10,000 times in different array sizes, we selected three types: $(10,10),(30,30)$, and (60, 60). They are a good representation of different types of indoor workplaces. Then, we selected a combination of parameter values that display the most significant and interesting behaviors. In particular, we showed the following combination of parameters: (S1) daily test and $90 \%$ of random sample, (S2) daily test and 75\% of random sample, and (S3) twice a week and 90\% of random sample. Based on this model specification, we present the dynamics of infected cells and the number of days-time steps-to control and stop the virus transmission (Figure 3).

Figure 3 illustrates the rapid decrease in days of infected cells using the (s1) and (s2) testing-isolating strategies. On the other hand, the strategy (s3) shows a gradual decrease in days of infected cells. Therefore, the daily testing interval is preferable 


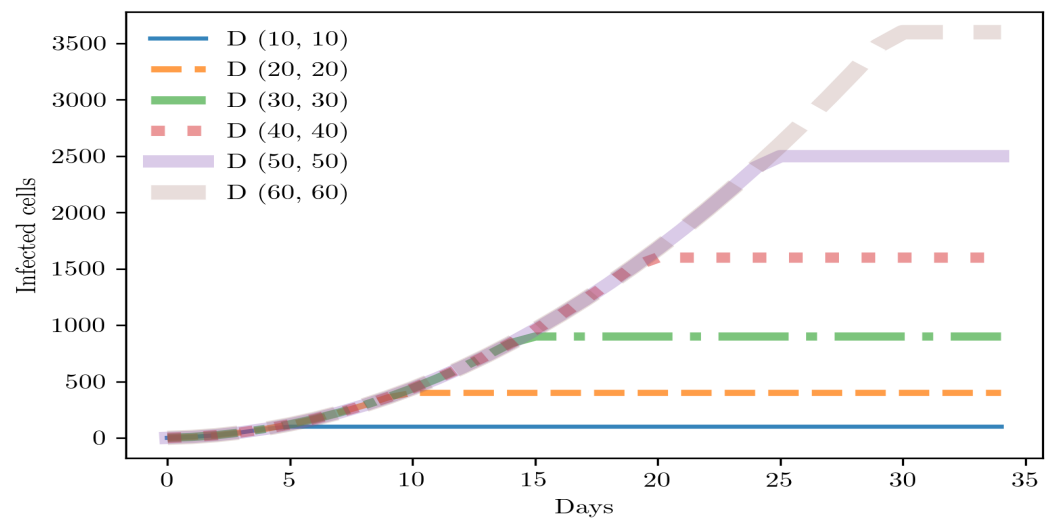

Figure 1. Virus transmission without any intervention in different array sizes. $D()$ represents the dimension of the array. The starting point of lines with zero slopes describes the number of days in which all cells were infected.

than the twice a week. The intensity of the test assists to identify faster infected cells. If the frequency of testing decreases, the identification of infected cells is slower and potentially harmful.

Next, we show findings associated with the sensitivity analysis based on scatter plots. We started with the array size of $(10,10)$ and display the three testing strategies.

Subfigure 4(a) shows the best combination of parameters to control the virus transmission in local indoor places. The daily testing and $90 \%$ of random sample controls the transmission in 2 to 9 days. Its Spearman coefficient suggests a high and positive correlation between these parameters. Subfigure 4(b) shows the second best strategy, twice a week testing and $75 \%$ of random sample. It controls the spread of disease in 2 to 17 days. Its Spearman coefficient is lower than, and the scatter points show higher dispersion than, the first strategy. Subfigure 4(c) shows the third strategy, daily testing and 75\% of random sample. This combination of parameters controls worse the transmission based on a large period of time. Its Spearman coefficient is lower than, and the scatter points show higher dispersion than, the first and second strategies. Finally, Subfigure 4(d) shows the statistical distributions and best fits based on the number of testing samples of each of the above strategies. The least expensive strategy is the daily testing and $75 \%$ of random sample because of the number of cell tested. It shows a median of 181.68 tested cells or in monetary terms between $\$ 218,016.0-\$ 1,362,600.0 \mathrm{mxn}(\$ 9,629.04-\$ 60,317.76$ usd) (Table 1$)$. The next least expensive is the daily testing and $90 \%$ of random sample. It shows a median of 194.47 tested cells or in monetary terms between $\$ 233,364.0-\$ 1,458,525.0 \mathrm{mxn}(\$ 10,306.9-\$ 64,564.0 \mathrm{usd})$. The most expensive strategy is twice a week and $90 \%$ of random sample. It shows a median of 284.24 tested cells or in monetary terms between $\$ 341,088.0-\$ 2,131,800.0 \mathrm{mxn}$ (\$15,064.7-\$94,367.6 usd).

After analyzing these findings, we concluded that the first strategy is the best one. It controls the virus transmission in at least one week and the cost of the testing is slightly higher than the least expensive strategy. Then, in this scale of analysis, we identify the combination of parameters presented in subfigure 4(a) as the most desirable intervention in small workplaces.

Next, we present the findings related to the array size of $(30,30)$ (Figure 5). Subfigure 5(a) shows the best combination of parameters to control the virus transmission in local indoor places. The daily testing and $90 \%$ of random sample controls the transmission in 2 to 11 days. Its Spearman coefficient suggests a high, positive correlation between these parameters. Subfigure 5(b) shows the second best strategy because it controls the spread of disease in 2 to more than 30 days-this is significantly higher than the past combination of parameters. Its Spearman coefficient is high and positive and suggests a strong correlation.

Subfigure 5(c) shows the third strategy in which the virus transmission was controlled on a larger period of time. Its Spearman coefficient is high and positive, and the scatter points show higher dispersion than the first and second strategies. Finally, Subfigure 5(d) identifies the least expensive strategy of the three. This strategy is the daily testing and $90 \%$ of random sample. It shows a median of 2028.41 tested cells or in monetary terms between $\$ 2,434,092.0-\$ 15,213,075.0 \mathrm{mxn}$ (\$107,505.7-\$673,432.1 usd) (Table 2).

After analyzing these findings, we concluded that the first strategy is the best one. It controls the virus transmission in at least one week and the cost of the testing is the least expensive one. Therefore, that combination of parameters suggested the most desirable intervention in medium-sized workplaces.

Finally, we present the findings related to the array size of $(60,60)$ (Figure 6). Subfigure 6(a) shows the best combination of parameters to control the virus transmission in local, but larger indoor places. The daily testing and $90 \%$ of random sample controls the transmission in approximately 15 days. Its Spearman coefficient suggests a high, positive correlation between these 
$t_{0}$
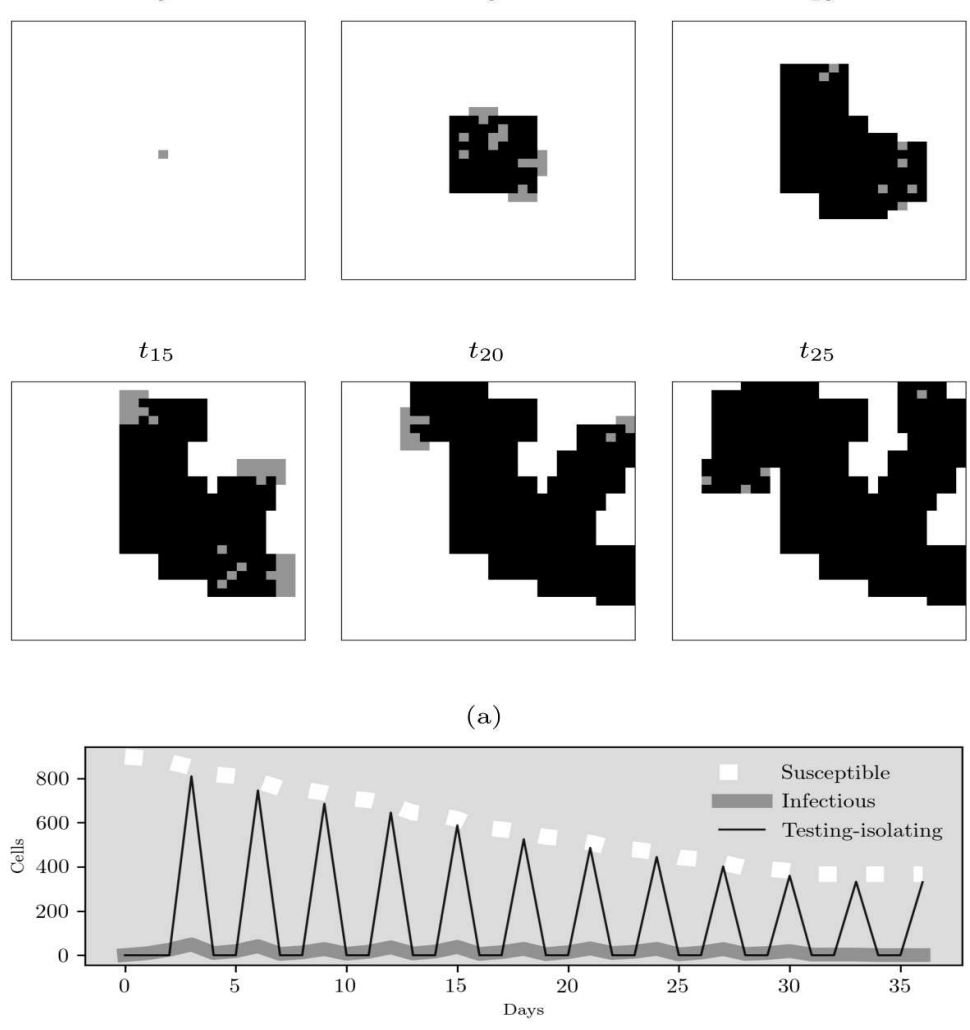

$t_{10}$
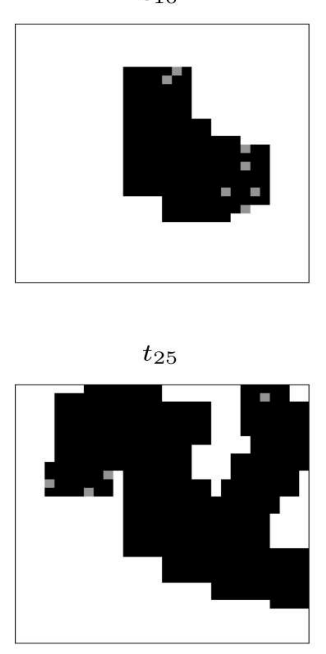

(a)

$t_{5}$

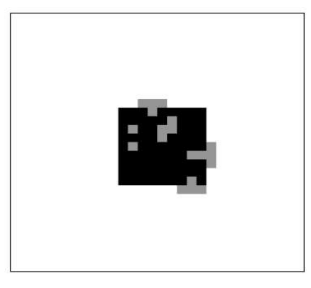

Figure 2. Dynamics of one CA simulation based on $\mathrm{D}(30,30)$, a twice a week frequency test, and $90 \%$ of tested people. The simulation stops when the transmission was controlled, i.e., the number of infected cells is equal to zero.

parameters. Subfigure 6(b) shows the second best strategy because it controls the spread of disease in more than 40 days. Its Spearman coefficient is high and positive suggesting a strong correlation. Subfigure 6(c) shows the third strategy in which the virus transmission was controlled in a larger period of time. Its Spearman coefficient is high and positive, and the scatter points show higher dispersion than the first and second strategies. Finally, Subfigure 6(d) identifies the least expensive strategy of the three. This strategy is the daily testing and $90 \%$ of random sample. It shows a median of 14466.10 tested cells or in monetary terms between $\$ 17,359,320.0-\$ 108,495,750.0 \mathrm{mxn}$ (\$766,703.3-\$4,802,745.2 usd) (Table 3).

These findings suggested that the first strategy is the best. It controls the virus transmission in around two weeks and the cost of the testing is the least expensive. Therefore, the combination of those parameters suggested the most desirable intervention in larger workplaces.

In summary, results showed that the best strategy in each of the array sizes is the combination of daily testing and $90 \%$ of random sample. This strategy shows the least expensive testing intervention, but these costs are significantly higher than any cost that we have known. The impact of these findings is that the virus transmission can be controlled in local indoor workplaces. This local strategy can be replicated to urban areas covering the most vulnerable locations; for example communities of the most vulnerable individuals due to comorbidities, age, race, or income. Then, we can better organize the return to different activities based on a credible and intelligent testing approximation for saving lives and reactivating the economy.

\section{Discussion}

Different interventions to reduce the SARS-CoV-2 transmission have been suggested by policy-makers around the world. Most of the time, these interventions have been ineffective. The main issue with a successful intervention is to generate a feasible and credible national strategy, based on science, where local communities and workplaces actively participate.

In this study, we proposed a local testing-isolating intervention for reducing the virus transmission in indoor workplaces to reopen and activate the economic system. Our formulation, based on the 2D CA and calibrated by official data of the Mexican epidemic, describes different scenarios that identify the most desirable testing time frequency and the most adequate random trails. However, these scenarios were not in line with our hypothesis. We expected that the time frequency of testing and the 
(a)
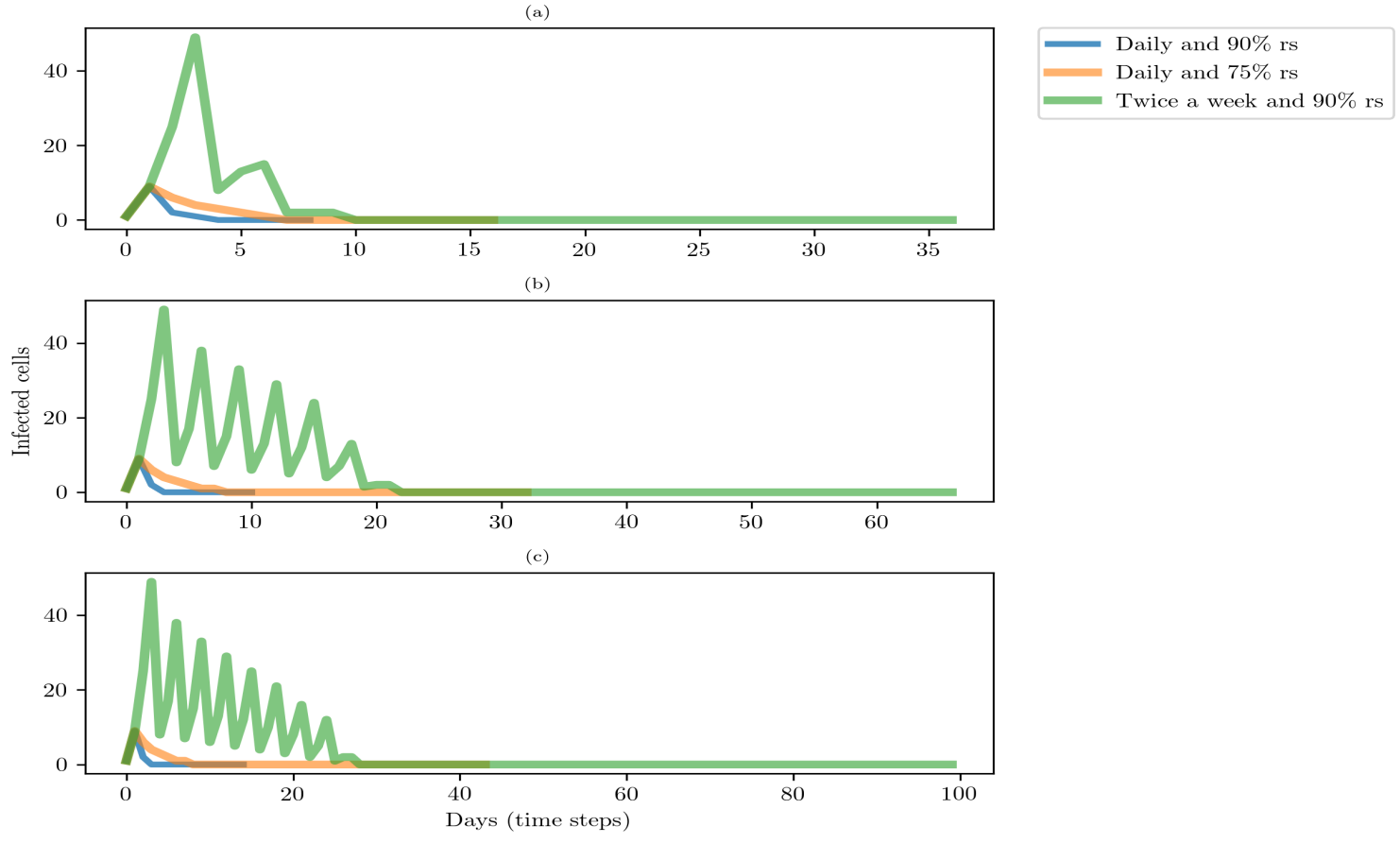

Figure 3. Trajectories of infected cells and number of days to control and stop the virus transmission. The computation of trajectories was based on the median of 10000 model executions. (a) Array size of $(10,10)$, (b) array size of $(30,30)$, and (c) array size of $(60,60)$. Percentage of random sample (\% rs).

size of random trails depended on the size of the array, but they do not. Moreover, we believed that a value of $70 \%$ of people in random trails was enough to mitigate the virus transmission, but it is not. We were right about the economic cost of the testing strategies. Therefore, we found that, in all cases of array sizes, the best time frequency is daily and the best percentage of sampling is at least $90 \%$. This combination of parameter values controls and stops the virus transmission in approximately a week. Compared with other scenarios, the economic cost is lower because the testing strategy is intensive in days and in size of random samples. The second best strategy is to apply the daily frequency and $75 \%$ of random samples. This strategy controls and stops the transmission in larger time steps and shows a larger number of infected cells before stoping the transmission. The economic cost is higher because the intensity of the testing strategy is in more time steps. Finally, the third strategy is related to the time frequency of twice a week and $90 \%$ of random samples. It is the most expensive strategy and the number of infected cells can be the total before controlling the transmission. If the goal of the strategy is to control and stop the spread of the virus, the testing time interval has not to be larger than twice a week.

We surprisingly identify that the economic cost of any intervention for controlling the SARS-CoV-2 transmission-or any other disease in the future-without a vaccine is higher than any other that we have known. Without a national plan that considers local communities and workplaces as the key to mitigate and decrease the virus transmission, we are doomed to repeat current mistakes. In particular, to establish the national health and the economic systems on political guidelines instead of science principles is a significant mistake that will accumulate overspending and lost of lives. Therefore, we suggest that our priorities have to be reorganized, where the health and the natural environment are above the economic interests.

Our results are encouraging and should be validated in real local communities and workplaces not only in Mexico, but also in other countries. We believe that an interdisciplinary approximation is appropriated to face the current health and economic costs. Therefore, we propose to apply the most intensive testing strategy to reopen the economy the safest way possible, minimizing the human cost, even if the economic cost is not the lowest. Therefore, the strategy we propose is the following:

1. The federal government has to invest in generating and distributing an efficient viral test. In particular, a test with less than $15 \%$ false positive and faster diagnose time, around $15 \mathrm{~min}$.

2. To reopen activities, first responders and essential workers have to be grouped based on the physical installations they share for work. For example, healthcare workers are grouped based on the building they continuously use through the day. Then, each group of first responders has to be tested daily and at least $90 \%$ of its population.

3. Regardless if all tested negative or not for the virus, given that the probability to get infected and spread the virus does not 

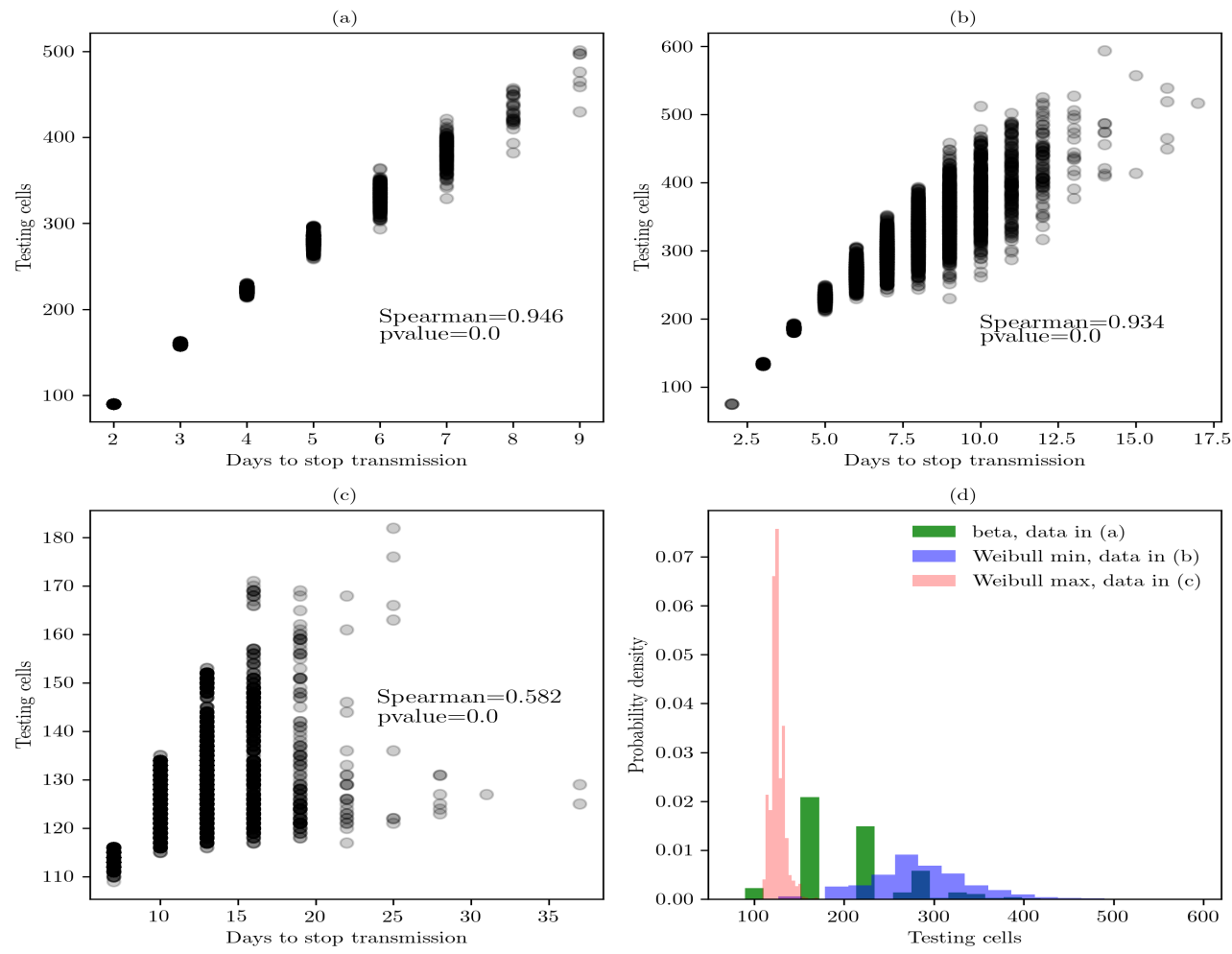

Figure 4. Sensitivity analysis based on scatterplots in the array of $(10,10)$. (a) Daily testing and $90 \%$ of random sample. (b) Twice a week testing and $90 \%$ of random sample. (c) Daily testing and $75 \%$ of random sample. (d) Distribution fitting of the cost for the testing strategy in the three cases. Data in subfigure (a) is best described by a beta distribution that shows a probability density function of the form $f(x, a, b)=\frac{\gamma(a+b) x^{\alpha-1}(1-x)^{b-1}}{\gamma(a) \gamma(b)}$, for $0<x<1, a>0, b>0$. Data in subfigure (b) is best described by a Weibull min distribution that shows a probability density function of the form $f(x, c)=c x^{c-1} \exp \left(-x^{c}\right)$, for $x \geq 0, c>0$. Data associated with (c) is best described by a Weibull max distribution that shows a probability density function of the form $f(x, c)=c(-x)^{c-1} \exp \left(-(-x)^{c}\right)$, for $x<0$ and $c>0$.

depend on whether a person tested negative a week before, and given that the symptoms may appear within 14 days, we suggest to wait one week to start the testing-isolating strategy again for at least $90 \%$ of the population chosen randomly at a daily frequency.

4. The testing strategy needs to continue until a vaccine can be distributed and applied for the population.

Finally, who is going to pay for the testing strategies? Each of us has to pay for it. The federal government has the constitutional obligation to give free access to any citizen of the healthcare system. Then, a revenue stream of the public sector has to pay at least $3 / 4$ of the total cost of the test, and let the society coordinate its implementation and monitoring. The responsibility of the government is to provide and distribute an efficient test, to remove any type of bureaucratic and political barriers to distribute such a test, and to let the science guide us through the epidemic. However, by looking at official records on deaths and the need to convert hospitals designated to fight other type of diseases and illness for COVID-19, it is evident that the current inaction of the government exemplifies the failure of different prevention policies through time. It is not enough that the government focuses on mitigating the epidemic; science has to guide the path towards actually fighting the virus and stopping the spread. On the other hand, as society, we have the responsibility to face and answer unresolved mistakes. If we start late to make actions for future pandemics, we will accumulate costs sooner rather than latter. Therefore, we have to rethink and generate our prevention strategies in which science will lead us.

\section{Conclusion}

Any type of intervention for controlling the SARS-CoV-2 transmission without a vaccine is extremely costly and challenging. Small-scale interventions are the best option because local communities can self-organize the testing and monitor infected cases. Local communities are the key to propagate the testing-isolating strategy at different scales. Therefore, first responders 

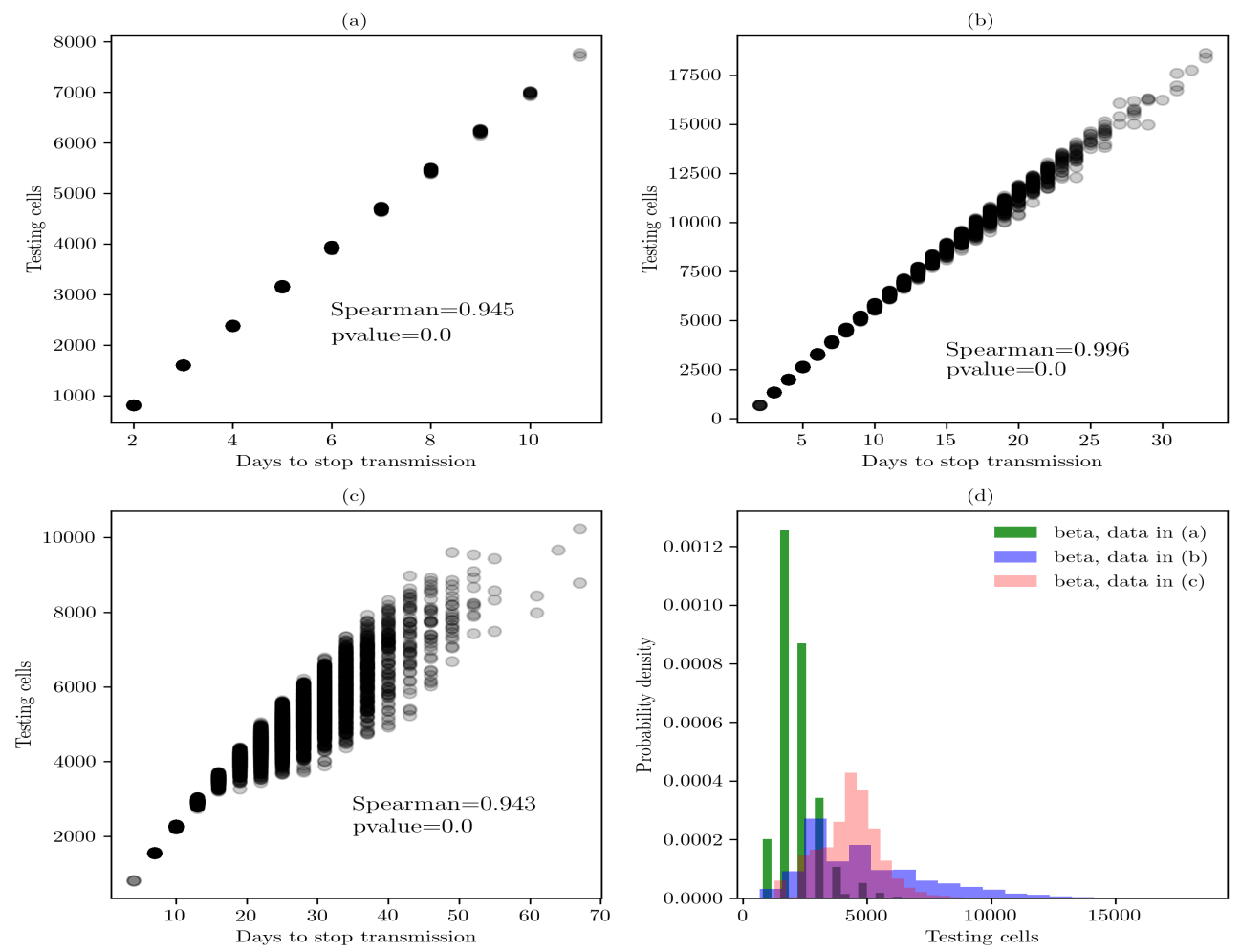

Figure 5. Sensitivity analysis based on scatterplots in the array of $(30,30)$. (a) Daily testing and $90 \%$ of random sample. (b) Twice a week testing and $90 \%$ of random sample. (c) Daily testing and $75 \%$ of random sample. (d) Distribution fitting of the cost for the testing strategy in the three cases. Data in (a), (b), and (c) are best described by a beta distribution that shows a probability density function of the form $f(x, a, b)=\frac{\gamma(a+b) x^{\alpha-1}(1-x)^{b-1}}{\gamma(a) \gamma(b)}$, for $0<x<1, a>0, b>0$.

and essential workers are the basic elements to initiate waves of testing-isolating interventions that control the virus until a vaccine is deployed to everyone.

To honor and remember those people who lost their lives due to the covid-19, we have to work closely with the scientific community for providing better-informed strategies to decision-makers and to coordinate global efforts for developing a solution for this unprecedented health and economic crisis.

\section{Data Availability}

The source code and the material and findings data of this study are openly available in the OSF, project Local testing SARS-CoV2 strategies and reopening the economy, at https://osf.io/7e98t/?view_only=6237b3c6bca 944 a 8b $18 \mathrm{~b} 0$ e $75 \mathrm{fa} 3 \mathrm{fb} 70 \mathrm{a}$ [DOI 10.17605/OSF.IO/7E98T].

\section{References}

1. Lewis, M. The economics of epidemics. Georgetown Journal of International Affairs 2, 25-31 (2001).

2. Dimitri, N. The economics of epidemic diseases. PLoS ONE 10, e0137964 (2015).

3. Bar-Yam, Y. Transition to extinction: Pandemics in a connected world. New England Complex Systems Institute (NECSI) (2016). URL https://necsi.edu/transition-to-extinction. (Accessed: Jun 29, 2020).

4. US Bureau of Labor and Statistics. The employment situation-may 2020. https://www.bls.gov/news.release/empsit.nr0.htm (2020). URL https://www.bls.gov/news.release/pdf/empsit.pdf. (Accessed: Jun 27, 2020).

5. INEGI. Encuesta telefónica de ocupación y empleo (etoe) 2020. https://www.inegi.org.mx/investigacion/etoe/ (2020). URL https://www.inegi.org.mx/contenidos/investigacion/etoe/doc/presentacion_ resultados.pdf. (Accessed: Jun 27, 2020). 

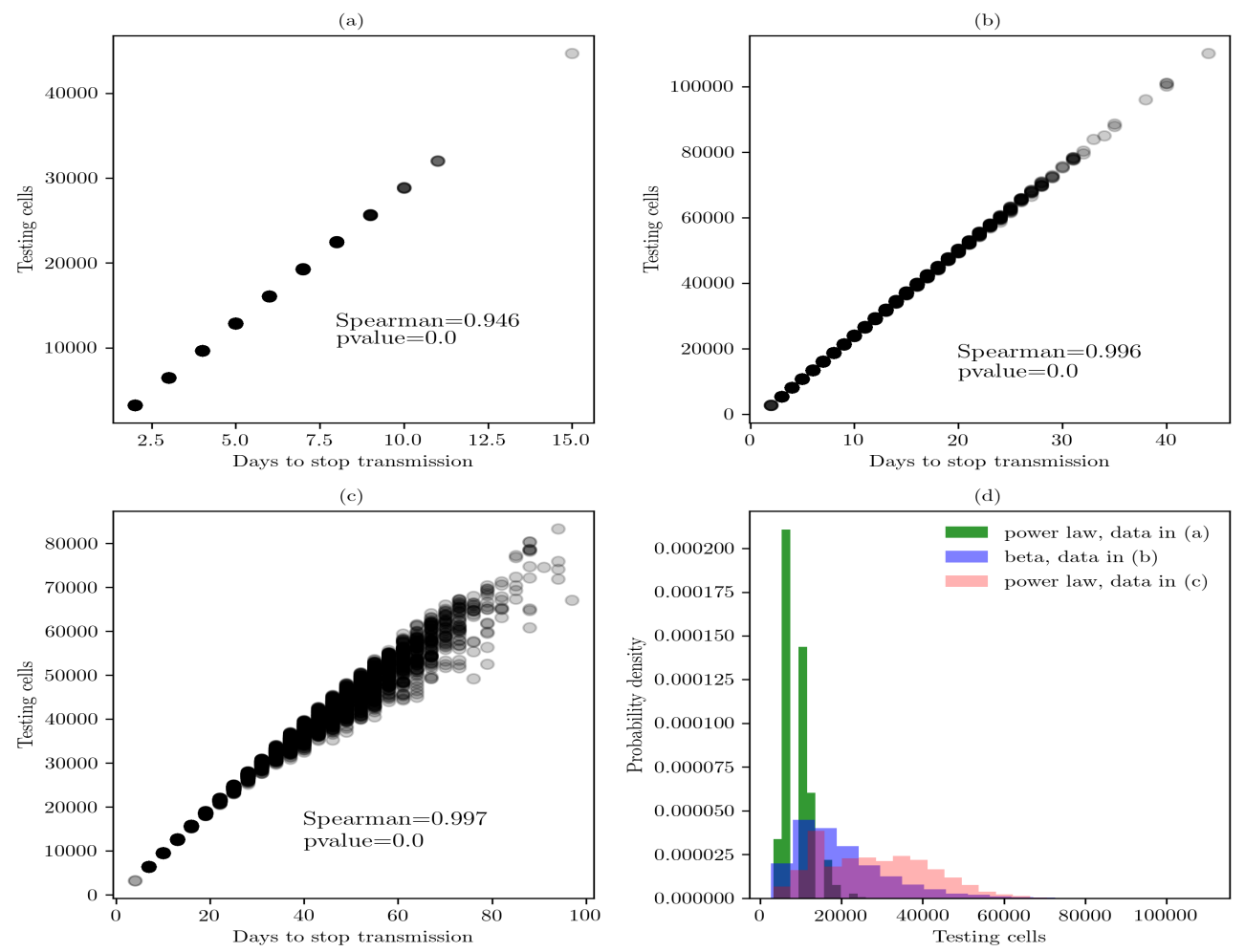

Figure 6. Sensitivity analysis based on scatterplots in the array of 60x60. (a) Daily testing-isolated and $90 \%$ of random sample. (b) Twice a week testing-isolated and $90 \%$ of random sample. (c) Daily testing-isolated and $75 \%$ of random sample. (d) Distribution fitting of the cost for the testing-isolated strategy in the three cases. Data in subfigures (a) and (c) is best described by a power law distribution that shows a probability density function of the form $f(x, \alpha)=\alpha x^{\alpha-1}$, for $0 \leq x \leq 1$, $\alpha>0$. Data in (b) is best described by a beta distribution that shows a probability density function of the form $f(x, a, b)=\frac{\gamma(a+b) x^{\alpha-1}(1-x)^{b-1}}{\gamma(a) \gamma(b)}$, for $0<x<1, a>0, b>0$.

6. CNN. Entire Dr. Anthony Fauci interview with Chris Cuomo. CNN Health (2020). URL https://edition.cnn.com/videos/health/2020/05/05/ cnngo-dr-anthony-fauci-chris-cuomo-covid-19-intv-cpt-sot.cnn. (Accessed: Jun 23, 2020).

7. Romer, P. Roadmap to responsibly reopen america. CNN Bussiness (2020). URL https://roadmap.paulromer. net/. (Accessed: May 10, 2020).

8. Osterholm, M. Episode 9: Smart testing. center for infectious disease research and policy (cidrap). University of Minessotta (2020). URL https: / / www. cidrap.umn. edu/covid-19/podcasts-webinars/episode-9. (Accessed: May 22, 2020).

9. CIDRAP. Part 3: Smart testing for covid-19 virus and antibodies. University of Minessotta (2020). URL https://www.cidrap.umn.edu/sites/default/files/public/downloads/ cidrap-covid19-viewpoint-part3.pdf. (Accessed: May 28, 2020).

10. Moreno, T. Científicos del cinvestav desarrollan prueba para detectar covid-19 en 15 minutos. El Universal (2020). URL https://www.eluniversal.com.mx/nacion/ cientificos-del-ipn-desarrollan-prueba-para-detectar-covid-19-en-15-minutos.

(Accessed: May 18, 2020).

11. Yu, L. et al. Rapid detection of covid-19 coronavirus using a reverse transcriptional loop-mediated isothermal amplification (rt-lamp) diagnostic platform. Clinical Chemistry hvaa102 (2020).

12. Toffoli, T. \& Margolus, N. Cellular Automata Machines: A New Environment for Modeling (The MIT Press, Cambridge, MA., 1987).

13. Wolfram, S. A New Kind of Science (Wolfram Media, 2002). 
14. Batty, M. Cities and Complexity: Understanding Cities with Cellular Automata, Agent-Based Models, and Fractals (The MIT Press, 2005).

15. Murray, J. Mathematical Biology (Springer-Verlag Berlin Heidelberg, 1989).

16. Allen, L. Some discrete-time si, sir, and sis epidemic models. Mathematical Biosciences 124, 83-105 (1994).

17. Hoya White, S., Martín del Rey, A. \& Rodríguez Sanchez, G. Modeling epidemics using cellular automata. Applied Mathematics and Computation 186, 93-202 (2007).

18. Rivers, M. Mass testing won't happen in mexico. that's the way the government wants it. CNN Bussiness (2020). URL https://edition.cnn.com/2020/05/15/americas/mexico-coronavirus-testing-intl/ index.html. (Accessed: May 22, 2020).

19. Gobierno de Mexico. Conferencias 25 de junio. https://coronavirus.gob.mx/2020/06/22/conferencia-25-de-junio/ (2020). URL coronavirus.gob.mx. (Accessed: Jun 26, 2020).

20. Gobierno de Mexico. Expertos de méxico, ops y el cdc participan en seminario sobre vigilancia epidemiológica y pruebas de diagnóstico. coronavirus.gob.mx (2020). URL https://coronavirus.gob.mx/2020/06/19/ expertos-de-mexico-ops-y-el-cdc-participan-en-seminario-sobre-vigilancia-epidemiologica-y (Accessed: July 02, 2020).

21. Siegenfeld, A. F., Taleb, N. N. \& Bar-Yam, Y. Opinion: What models can and cannot tell us about covid-19. Proceedings of the National Academy of Sciences (2020). URL https://www . pnas.org/content/early/2020/06/23/ 2011542117. https://www.pnas.org/content/early/2020/06/23/2011542117.full.pdf.

22. Nosek, B. A. et al. Promoting an open research culture. Science 348, 1422-1425 (2015).

23. Prather, K., Wang, C. \& Schooley, R. Reducing transmission of sars-cov-2. Science eabc6197 (2020).

24. Zhang, R., Li, Y., Zhang, A., Wang, Y. \& Molina, M. Identifying airborne transmission as the dominant route for the spread of covid-19. PNAS (2020).

25. Dbouka, T. \& Drikakisb, D. On coughing and airborne droplet transmission to humans. Physics of Fluids 32 (2020).

26. Organización Panamericana de Salud. Similitudes y diferencias entre la covid-19 y la gripe. OPS (2020). URL https://www.paho.org/hq/index.php?option=com_content\&view=article\&id=15760: similarities-and-differences-covid-19-and-influenza\&Itemid=1926\&lang=es. (Accessed: Jun 13, 2020).

27. Gobierno de Mexico. Conferencias 22 de junio. https://coronavirus.gob.mx/2020/06/22/conferencia-22-de-junio/ (2020). URL coronavirus. gob.mx. (Accessed: Jun 23, 2020).

28. Haushofer, J. \& Metcalf, C. Which intervention work best in a pandemic? Science 368, 1063-1065 (2020).

29. Rileya, S., Eamesb, K., Ishamc, V., Mollisond, D. \& Trapmane, P. Five challenges for spatial epidemic models. Epidemics 10, 68-71 (2015).

30. Heesterbeek, H. et al. Modeling infectious disease dynamics in the complex landscape of global health. Science $\mathbf{3 4 7}$ (2015).

31. Walker, P. G. T. et al. The impact of covid-19 and strategies for mitigation and suppression in low- and middle-income countries. Science (2020).

32. Massey, F. The kolmogorov-smirnov test for goodness of fit. Journal of the American Statistical Association 46, 68-78 (2020).

\section{Author contributions statement}

I.L. and M.G.A-C conceived the original idea. I.L. designed the method and the computational framework. I.L. and M.G.A-C analyzed the data and contributed to the final version of the manuscript.

\section{Ethics declarations}

Competing interests. The authors have declared that no competing interests exist. 


\begin{tabular}{|l|l|l|l|l|l|l|}
\hline $\mathrm{n}$ & Test interval & \% rs & Best fit & Parameters & KS test & First moments \\
\hline 10,000 & Daily & 90 & Beta & $(9.0898$, & $(0.0050$, & $(199.4736$, \\
& & & & 2598074.4982, & $0.9629)$ & 206.3408, \\
& & & & 17.7949, & & 3910.8787, \\
& & & & $53890486.4759)$ & & 0.6633, \\
& & & & $0.6600)$ \\
\hline 10,000 & Daily & 75 & Weibull Min & $(106.2499$, & $(0.0063$, & $(284.2427$, \\
& & & & 2191.0265, & $0.8213)$ & 283.0571, \\
& & & & 325.4431, & & 3716.8704, \\
& & & & $13174.2205)$ & & -0.0636, \\
& & & & & $-0.2631)$ \\
\hline 10,000 & Twice a week & 90 & Weibull Max & $(0.2251$, & $(0.0042$, & $(181.6816$, \\
& & & & 182.00, & $0.9930)$ & 104.7816, \\
& & & & $1.6213)$ & & 726484.8260, \\
& & & & & & -99.6032, \\
& & & & & & $42214.9272)$ \\
\hline
\end{tabular}

Table 1. Statistics of testing-isolated strategies in the array of $(10,10)$. Percentage of random sample $(\% \mathrm{rs})$. Estimated parameters (a, b, loc, scale). KS goodness-of-fit test (D, p-value). First moments (median, mean, variance, skewness, kurtosis).

\begin{tabular}{|l|l|l|l|l|l|l|}
\hline $\mathrm{n}$ & Test interval & \% rs & Best fit & Parameters & KS test & First moments \\
\hline 10,000 & Daily & 90 & Beta & $(2.8583$, & $(0.0060$, & $(2028.4100$, \\
& & & & 16.9468, & $0.8575)$ & 2164.2004, \\
& & & & 534.3316, & & 757008.4565, \\
& & & & $11293.0408)$ & & 0.8468, \\
& & & & & $0.7654)$ \\
\hline 10,000 & Daily & 75 & Beta & $(2.9444$, & $(0.0051$, & $(4503.4733$, \\
& & & 90294701.0929, & $0.9536)$ & 4988.4108, \\
& & & 605.5727, & & 6523948.4271, \\
& & & $134405606190.0885)$ & & 1.1655, \\
& & & & & $2.0377)$ \\
\hline 10,000 & Twice a week & 90 & Beta & $(1531.3427$, & $(0.0065$, & $(4309.6076$, \\
& & & & 60095.0441, & $0.7917)$ & 4319.5363, \\
& & & & -43679.9023, & & 1467117.2611, \\
& & & & $1931659.0267)$ & & 0.0491, \\
& & & & & & $0.0035)$ \\
\hline
\end{tabular}

Table 2. Statistics of testing-isolated strategies in the array of $(30,30)$. Percentage of random sample (\% rs). Estimated parameters (a, b, loc, scale). KS goodness-of-fit test (D, p-value). First moments (median, mean, variance, skewness, kurtosis). 


\begin{tabular}{|l|l|l|l|l|l|l|}
\hline $\mathrm{n}$ & Test interval & $\% \mathrm{rs}$ & Best fit & Parameters & KS test & First moments \\
\hline 10,000 & Daily & 90 & Power law & $(0.5275$, & $(0.0067$, & $(14466.1018$, \\
& & & & 3239.9999, & $0.7491)$ & 17665.0273, \\
& & & & $41768.5146)$ & & $1.56052589 \mathrm{e}+08$, \\
& & & & & & 0.5863, \\
& & & & $0.9234)$ \\
\hline 10,000 & Daily & 75 & Beta & $(2.9417$, & $(0.0066$, & $(19600.5595$, \\
& & & & 16.7101, & $0.7744)$ & 21199.0635, \\
& & & & 1169.8513, & & $1.10342031 \mathrm{e}+08$, \\
& & & & $133801.8123)$ & & 0.8243, \\
& & & & & $0.7094)$ \\
\hline 10,000 & Twice a week & 90 & Power law & $(0.6942$, & $(0.0066$, & $(35737.5789$, \\
& & & & 3239.9999, & $0.7653)$ & 39380.7156, \\
& & & & $88198.7279)$ & & $6.98309853 \mathrm{e}+08$, \\
& & & & & & 0.3260, \\
& & & & & $-1.1526)$ \\
\hline
\end{tabular}

Table 3. Statistics of testing-isolated strategies in the array of $(60,60)$. Percentage of random sample (\% rs). Estimated parameters (a, b, loc, scale). KS goodness-of-fit test (D, p-value). First moments (median, mean, variance, skewness, kurtosis). 
Figures

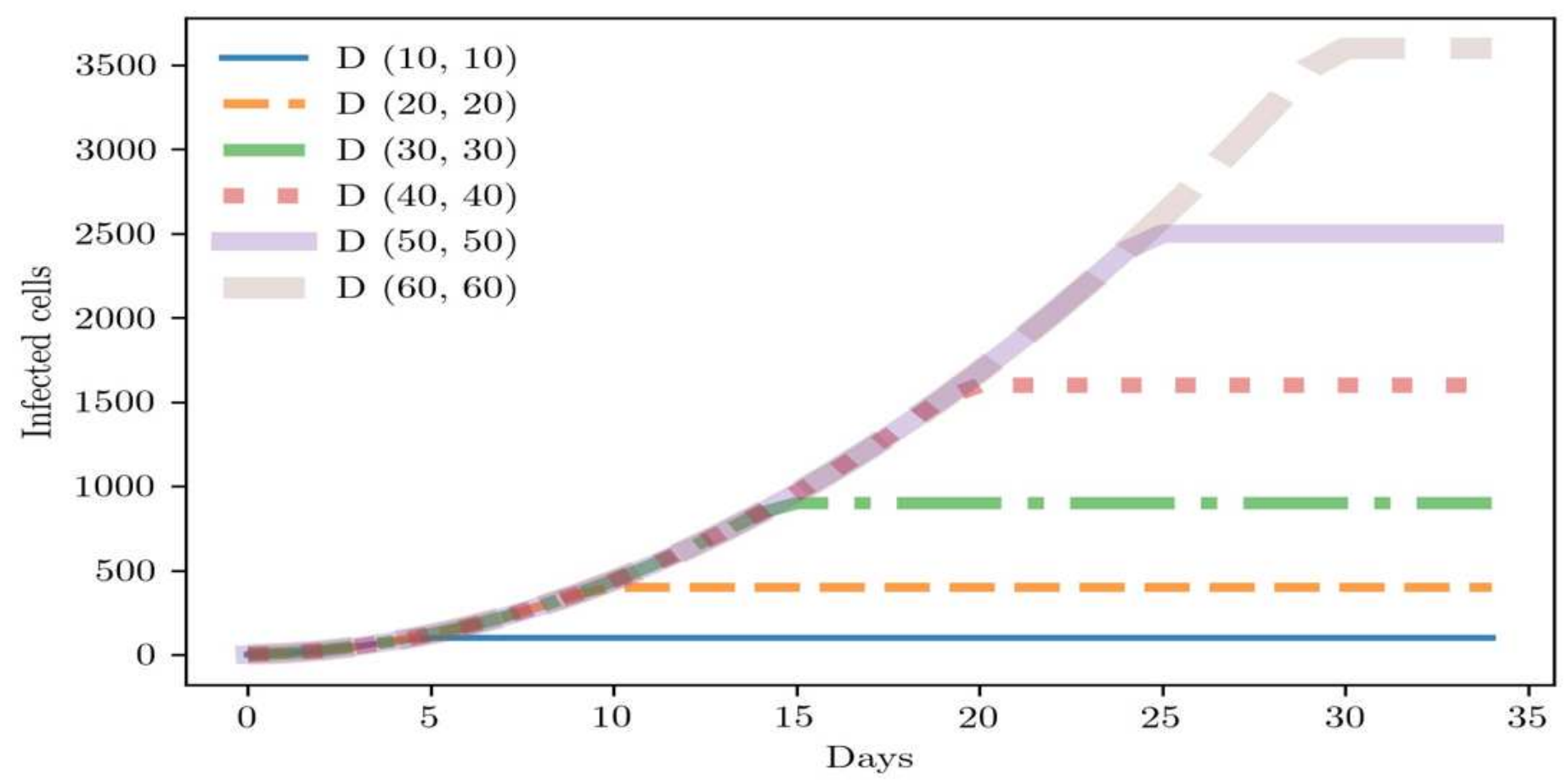

\section{Figure 1}

Virus transmission without any intervention in different array sizes. $D()$ represents the dimension of the array. The starting point of lines with zero slopes describes the number of days in which all cells were infected. 
$t_{0}$

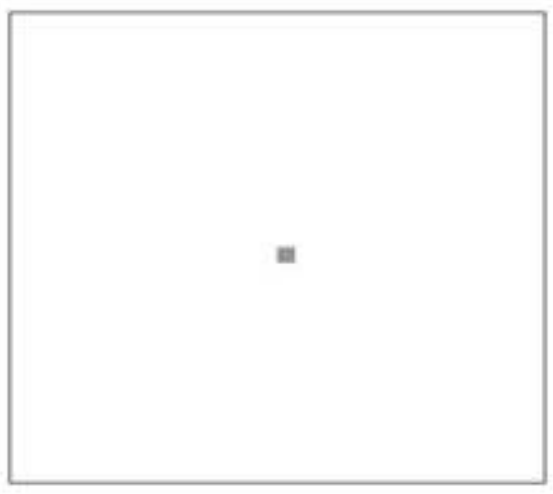

$t_{15}$

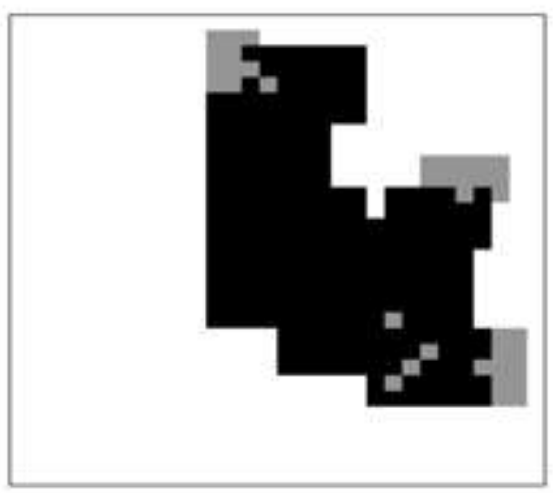

$t_{5}$

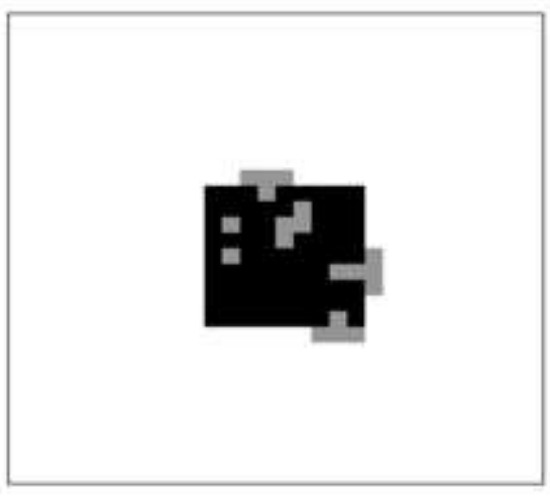

$t_{20}$

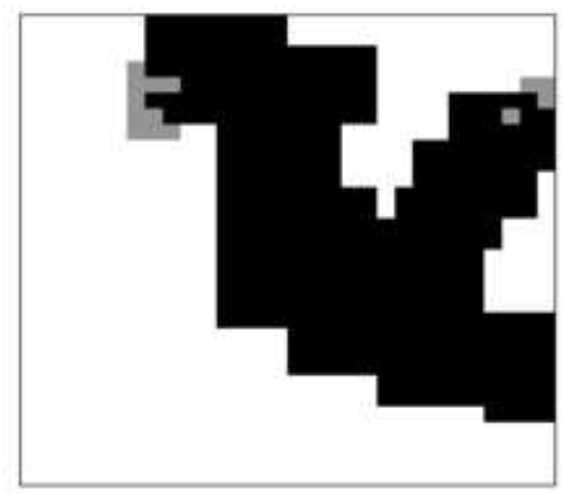

$t_{10}$

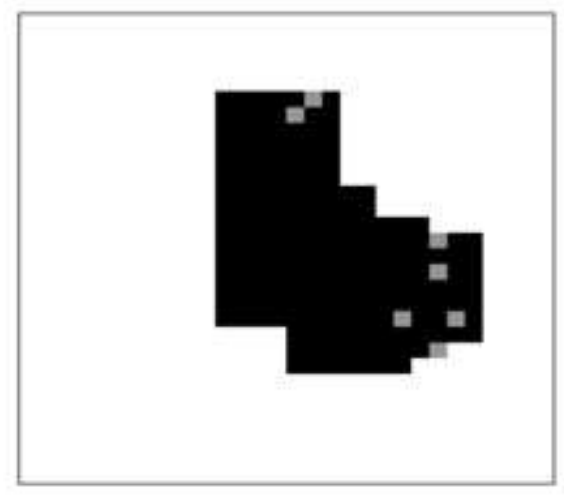

$t_{25}$

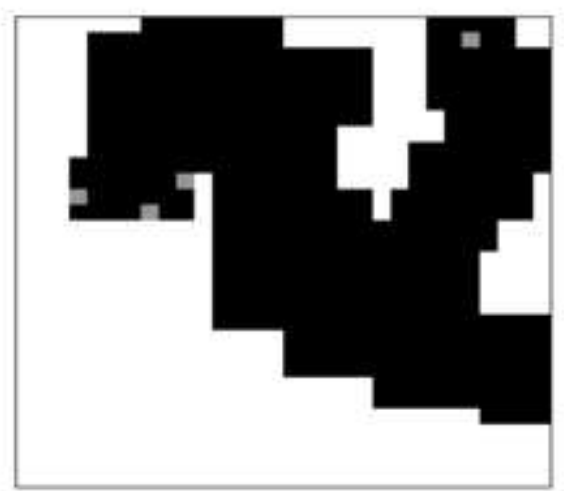

(a)

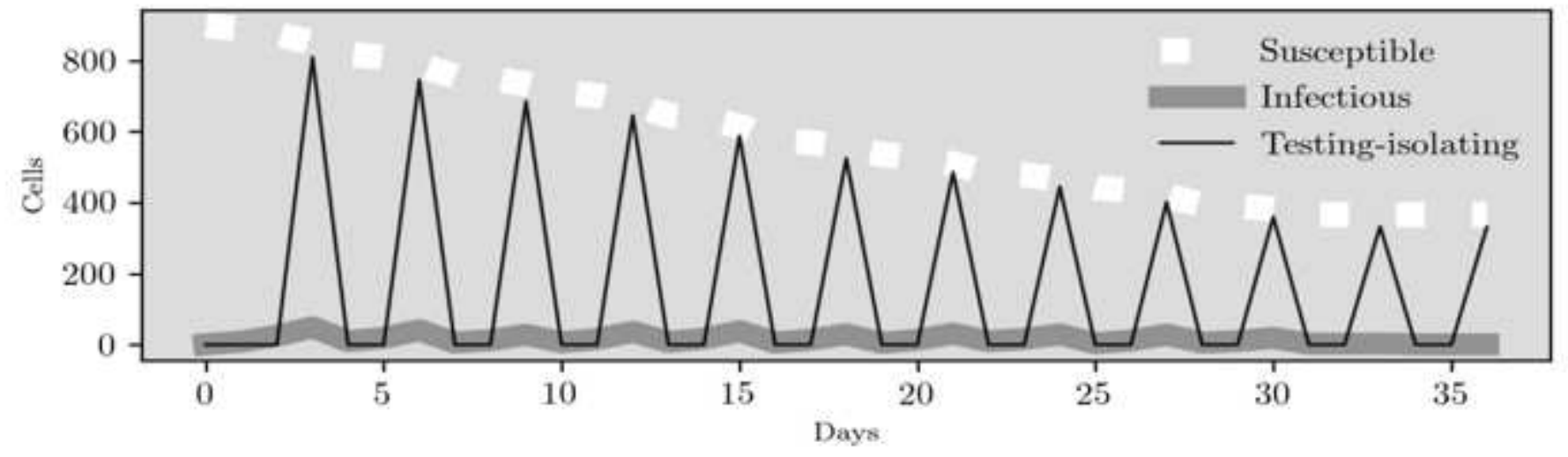

Figure 2

Dynamics of one CA simulation based on $\mathrm{D}(30,30)$, a twice a week frequency test, and $90 \%$ of tested people. The simulation stops when the transmission was controlled, i.e., the number of infected cells is equal to zero. 
(a)

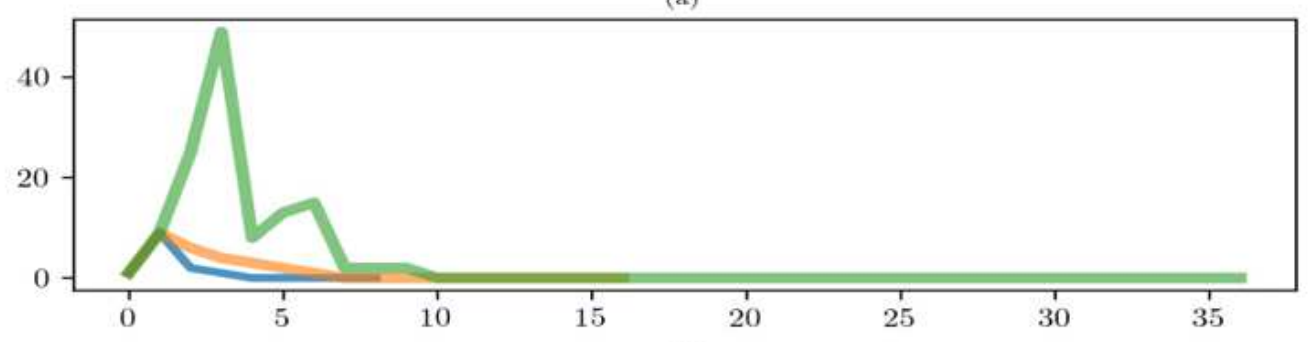

(b)

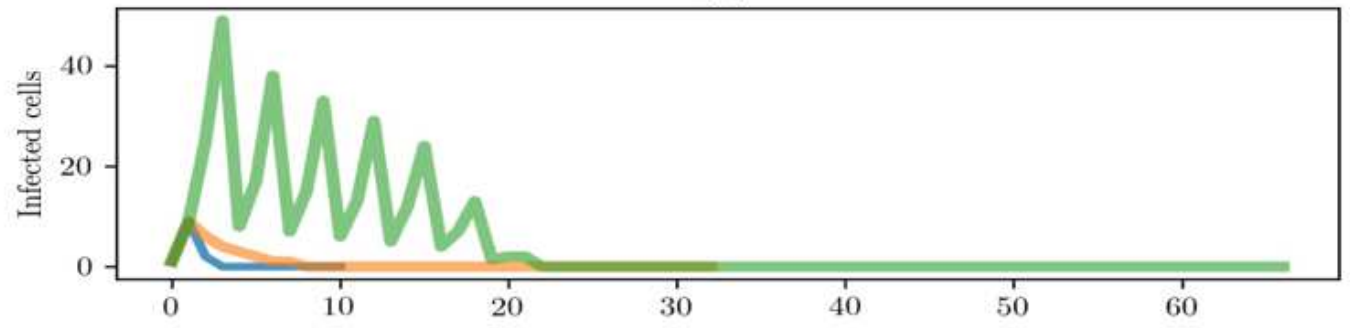

(c)

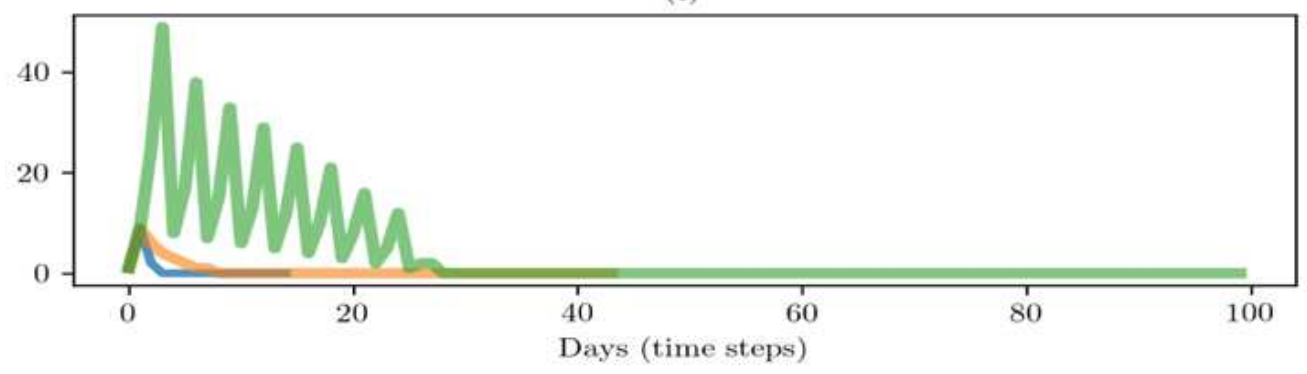

Daily and $90 \%$ rs

Daily and $75 \%$ rs

Twice a week and $90 \%$ rs

\section{Figure 3}

Trajectories of infected cells and number of days to control and stop the virus transmission. The computation of trajectories was based on the median of 10000 model executions. (a) Array size of $(10,10),(b)$ array size of $(30,30)$, and (c) array size of $(60,60)$. Percentage of random sample (\% rs). 
(a)

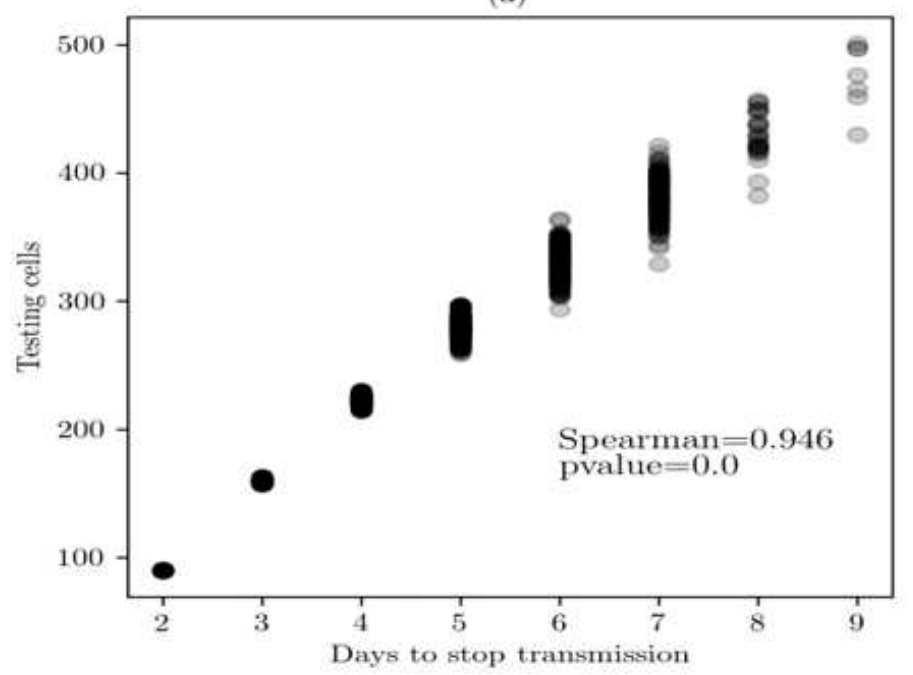

(c)

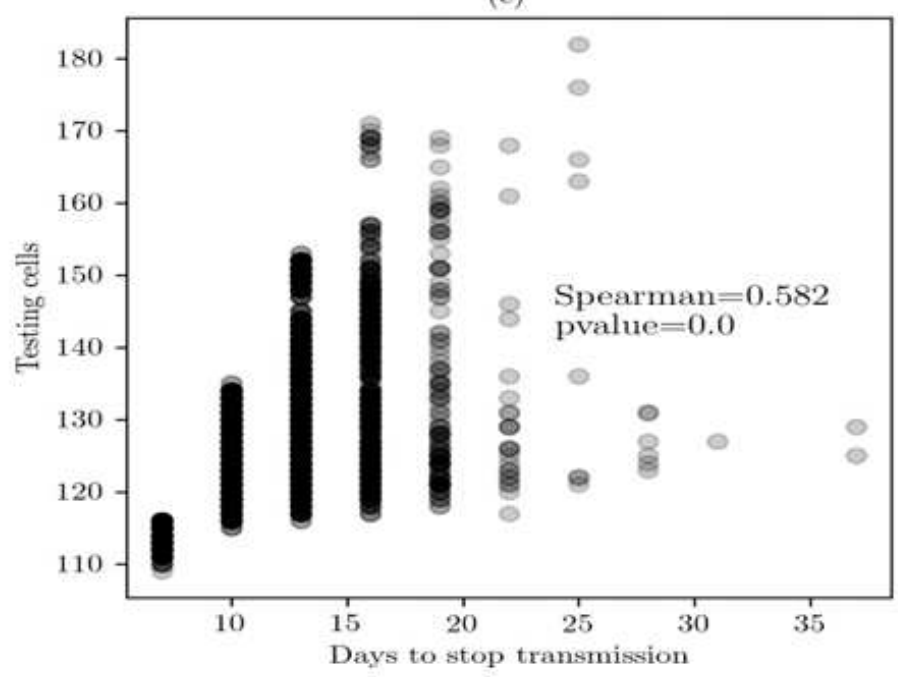

(b)

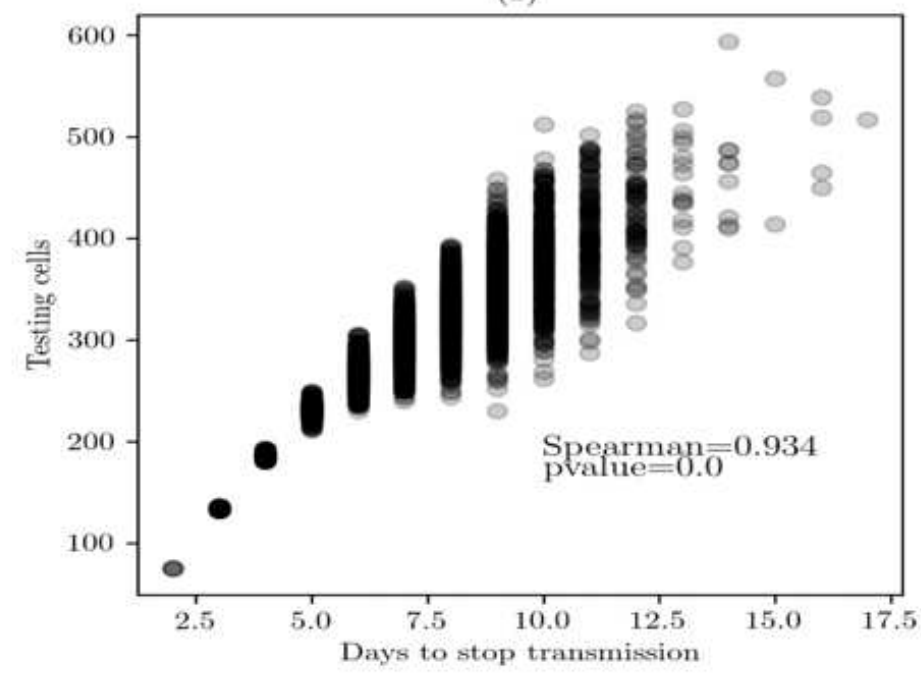

(d)

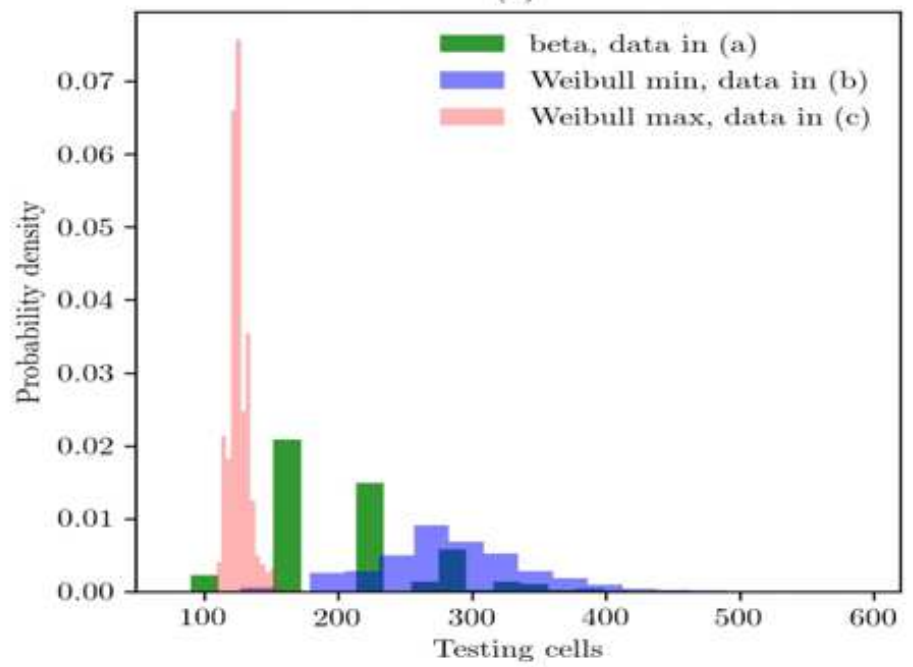

\section{Figure 4}

Sensitivity analysis based on scatterplots in the array of $(10,10)$. (a) Daily testing and $90 \%$ of random sample. (b) Twice a week testing and $90 \%$ of random sample. (c) Daily testing and $75 \%$ of random sample. (d) Distribution fitting of the cost for the testing strategy in the three cases. Data in subfigure (a) is best described by a beta distribution that shows a probability density function of the form $f(x, a, b)=$ $y(a+b) x a-1(1-x) b-1$, for $0<x<1, a>0, b>0$. Data in subfigure (b) is best described by a Weibull min distribution that shows a probability density function of the form $f(x, c)=c x c-1 \exp (-x c)$, for $x \geq 0, c>0$. Data associated with (c) is best described by a Weibull max distribution that shows a probability density function of the form $f(x, c)=c(-x) c-1 \exp (-(-x) c)$, for $x<0$ and $c>0$. 
(a)

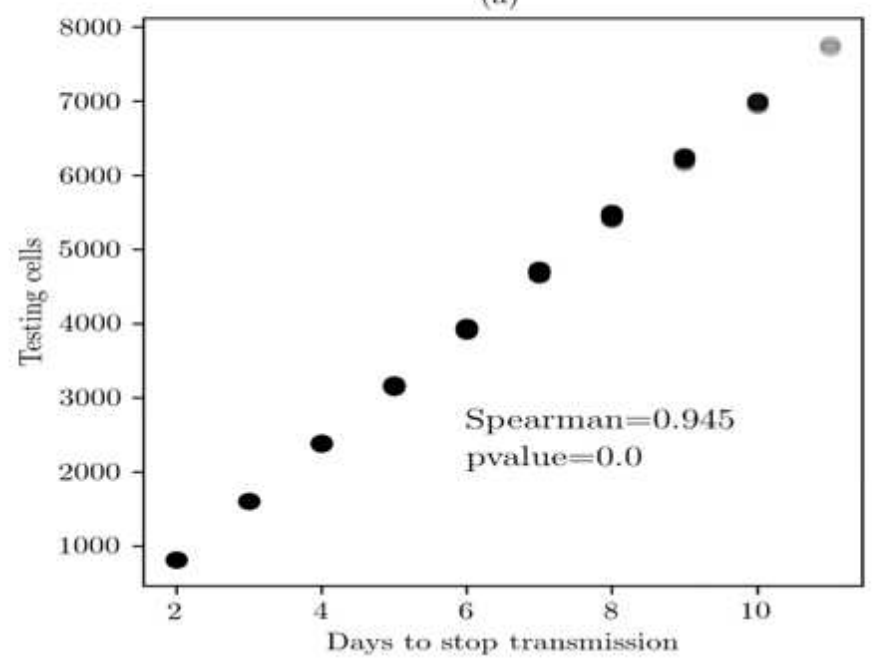

(c)

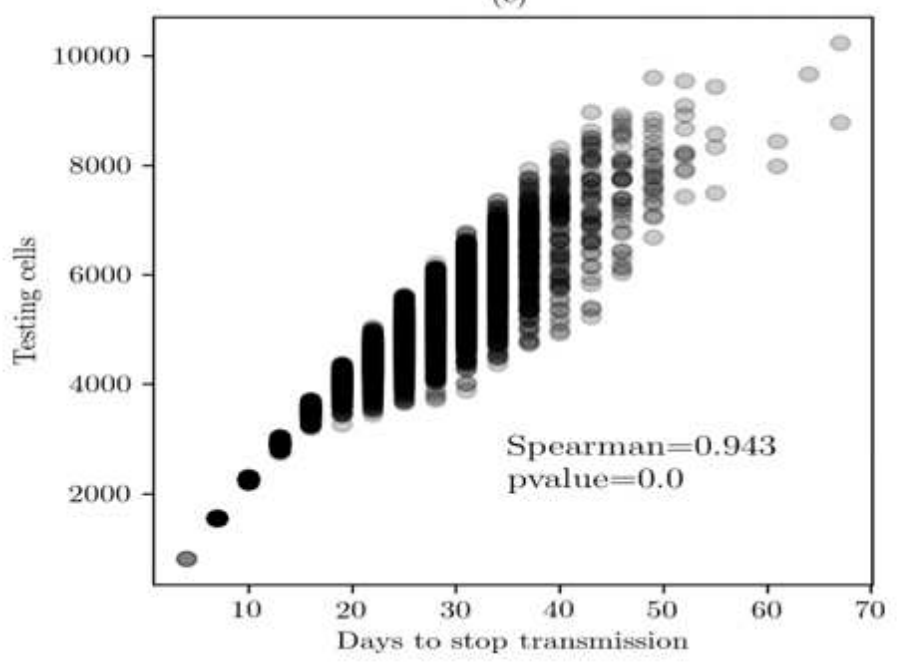

(b)

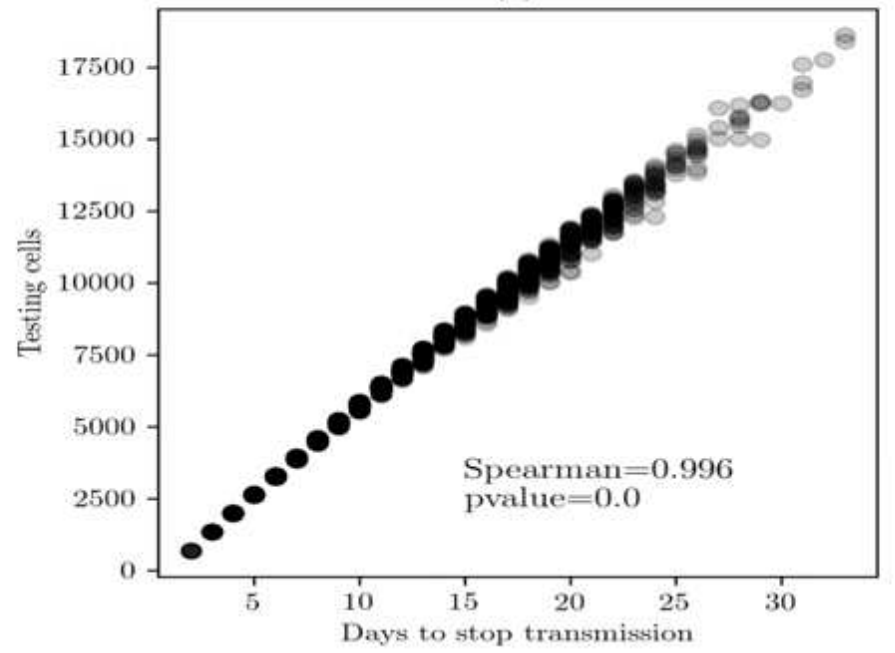

(d)

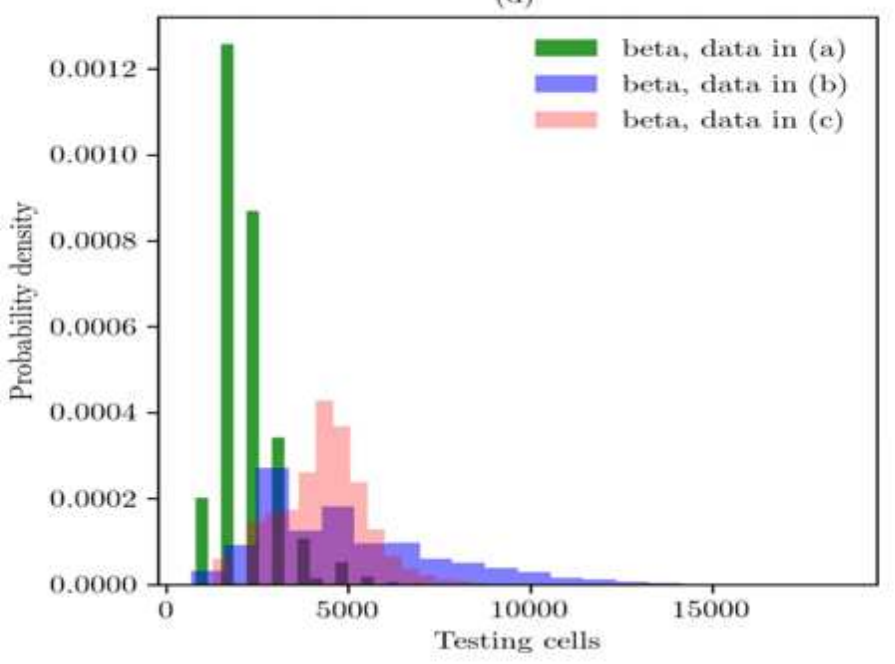

Figure 5

Sensitivity analysis based on scatterplots in the array of $(30,30)$. (a) Daily testing and $90 \%$ of random sample. (b) Twice a week testing and $90 \%$ of random sample. (c) Daily testing and $75 \%$ of random sample. (d) Distribution fitting of the cost for the testing strategy in the three cases. Data in (a), (b), and (c) are best described by a beta distribution that shows a probability density function of the form $f(x, a$, b) $=y(a+b) x a-1(1-x) b-1$, for $0<x<1, a>0, b>0$. 
(a)

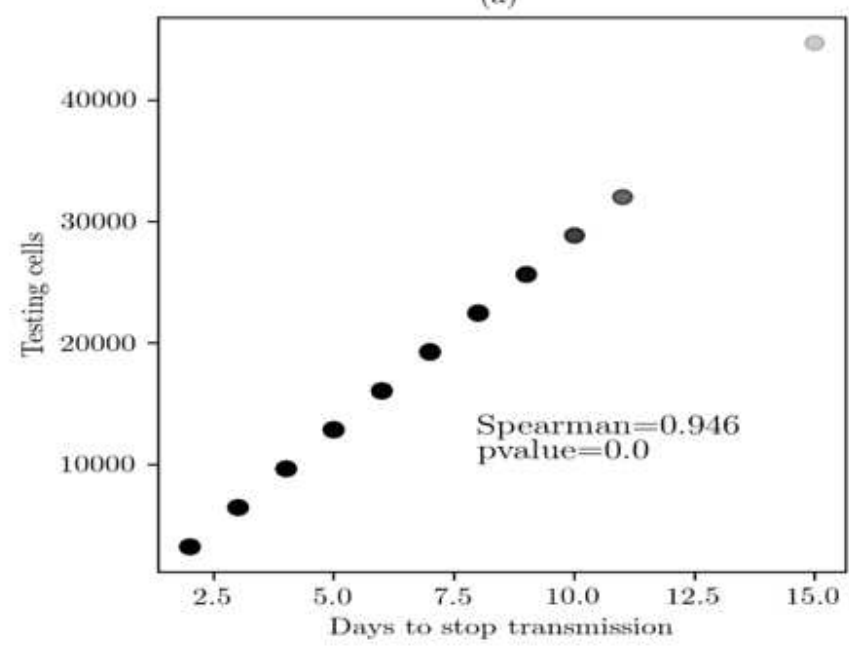

(c)

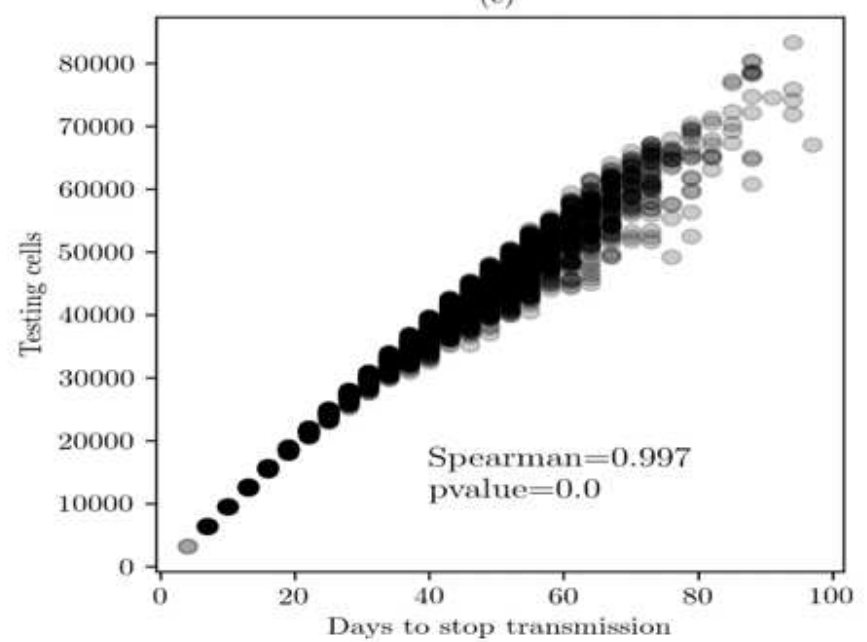

(b)

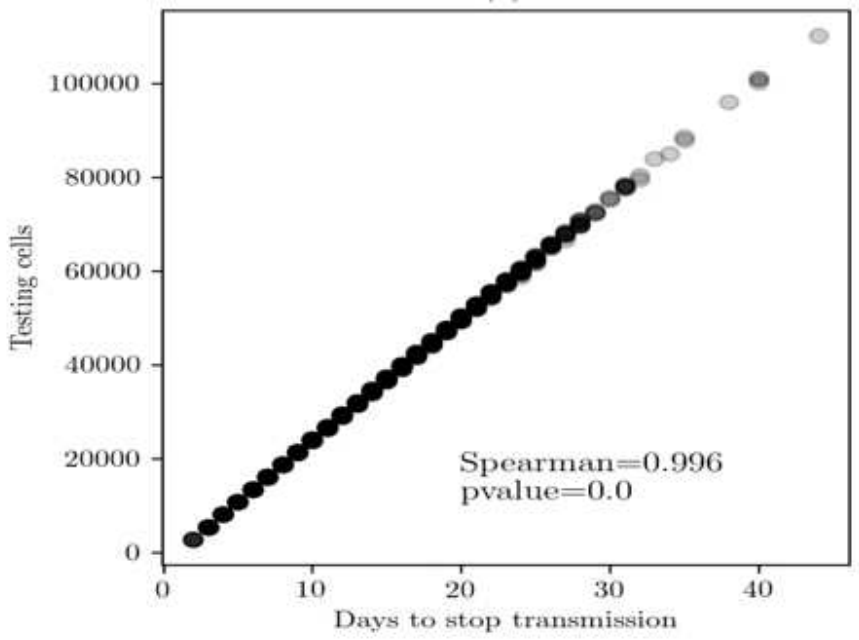

(d)

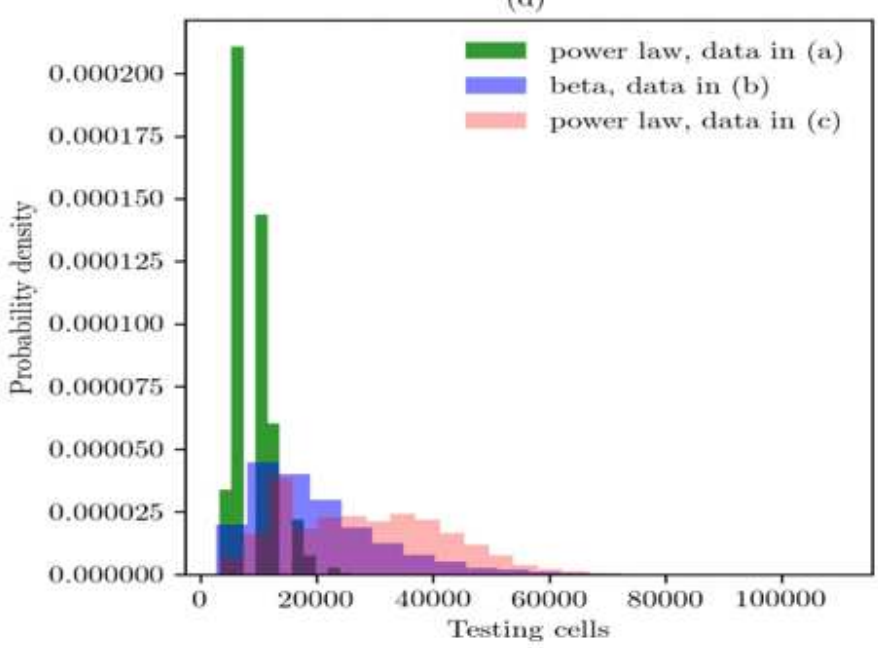

\section{Figure 6}

Sensitivity analysis based on scatterplots in the array of $60 \times 60$. (a) Daily testing-isolated and $90 \%$ of random sample. (b) Twice a week testing-isolated and $90 \%$ of random sample. (c) Daily testing-isolated and $75 \%$ of random sample. (d) Distribution fitting of the cost for the testing-isolated strategy in the three cases. Data in subfigures (a) and (c) is best described by a power law distribution that shows a probability density function of the form $f(x, a)=a x a-1$, for $0 \times 1, a>0$. Data in (b) is best described by a beta distribution that shows a probability density function of the form $f(x, a, b)=y(a+b) x a-1(1-x) b-1$, for $0<x<1, a>0, b>0$. 